DOI: 10.1136/annrheumdis-2017-eular.1695

\section{SAT0768-HPR SUBJECTIVE COGNITIVE DYSFUNCTION AND COMPUTERIZED NEUROPSYCHOLOGICAL PERFORMANCE IN KOREAN ADULTS WITH RHEUMATOID ARTHRITIS}

S.Y. Shin ${ }^{1}$, J.H. Lee ${ }^{2}$, B.Y. Yoon ${ }^{2} .{ }^{1}$ Department of Nursing, College of Medicine, Inje University; ${ }^{2}$ Division of Rheumatology, College of Medicine, Inje University, Ilsan Paik Hospital, Busan, Korea, Republic Of

Background: There is an increased appreciation of the burden of cognitive impairment in persons with rheumatoid arthritis (RA). Research shows a gap between subjective and objective measures of cognitive impairment in persons with chronic diseases.

Objectives: This study explored the relationship between subjective cognitive dysfunction and computerized neuropsychological performance in Korean older adults with RA.

Methods: Individuals with RA were recruited by their rheumatologists during follow-up visits at one university hospital in Korea. After getting signed consents, a trained research nurse assessed participants with a range of physical, psychosocial, and biological metrics. Subjective cognitive dysfunction was assessed using the Perceived Deficits Questionnaire (PDQ; range 0-20, higher score $=$ greater impairment). Objective cognitive impairment was assessed using a set of 6 computerized neurocognitive tests yielding 18 indices covering a range of cognitive domains. Subjects were classified as "impaired" if they performed $1 S D$ below age-based population norms on each test [1]. A total cognitive impairment score was calculated by summing the transformed scores (range: $0-18$, higher score=greater impairment). Multiple regression analysis controlling for education, disease severity, and depression was conducted to identify the relationship between objective and subjective cognitive measures.

Results: Fifty four subjects with a mean $( \pm S D)$ age of $63.6( \pm 10.5)$ years were included in the final analyses. $85 \%$ were female and $87 \%$ were married. Mean educational level was $10.2( \pm 4.9)$ years and mean disease duration was $8.9( \pm 8.5)$ years. $25.9 \%$ had depression and $55.6 \%$ had sleep problem. Mean PDQ score was 11.8 ( \pm 4.5 , range 5-25) and mean total cognitive impairment score was $11.0( \pm 4.1$, range $=2-18) .92 \%$ were classified as cognitively impaired on five or more test indices. There was no significant correlation between PDQ score and total cognitive function score $(r=.260, p=.068)$. However, psychological factors including depression $(\mathrm{r}=.621, p<.001)$ and sleep problem $(\mathrm{r}=.577, p<.001)$ were significantly correlated with $\mathrm{PDQ}$ score. In the multivariate analysis, there was no significant relationship between PDQ score and total cognitive impairment score. However, functional limitations and depression $(=0.317, p=0.048 ;=0.334$, $p=0.019$ ) were significantly associated with the $\mathrm{PDQ}$ score.

Conclusions: There was no significant relationship between subjective cognitive dysfunction and computerized neuropsychological performance in this cohort. Functional limitations and depression were significantly associated with perceived cognitive dysfunction. Findings emphasize the gap between subjective and objective measures of cognitive impairment and the importance of considering psychological factors in the context of cognitive complaints in clinical settings.

\section{References:}

[1] Kozora, E., Ellison, M. C., \& West, S. (2004). Reliability and validity of the proposed American College of Rheumatology neuropsychological battery for systemic lupus erythematosus. Arthritis Care \& Research, 51(5), 810-818.

Acknowledgements: This research was supported by the 2015 Inje University research grant (No.20151092).

Disclosure of Interest: None declared

DOI: 10.1136/annrheumdis-2017-eular.1299

\section{SAT0769-HPR DO PATIENTS RECALL PHYSICAL ACTIVITY ADVICE? INTERVIEWS WITH UK INFLAMMATORY ARTHRITIS PATIENTS TO EXPLORE EXPERIENCES OF PHYSICAL ACTIVITY COMMUNICATION}

V. Salmon ${ }^{1}$, N. Walsh ${ }^{2}$, E. Dures ${ }^{2}$, A. Haase ${ }^{3}$, M. Urban ${ }^{4}$, F. Cramp ${ }^{2}$. ${ }^{1}$ University of Exeter, Exeter; ${ }^{2}$ University of the West of England; ${ }^{3}$ University of Bristol; ${ }^{4}$ University Hospitals Bristol NHS Foundation Trust, Bristol, United Kingdom

Background: Guidelines for managing inflammatory arthritis (IA) indicate that patients should be encouraged to be physically active. Patient perceptions of physical activity $(\mathrm{PA})$ communication during routine rheumatology consultations, or whether they recall or understand PA information, are not known.

Objectives: 1. To explore patients' short-term recollection and interpretation of communication relating to PA during consultation with a rheumatology doctor or nurse

2. To explore patients' needs for effective PA communication

Methods: Adults with IA took part in semi-structured telephone interviews 2 to 3 days after their consultation with a rheumatology doctor or nurse. Transcripts were analysed using thematic analysis.

Results: 26 patients took part (18 female; age $35-83$ years; disease duration $0.25-40$ years). Preliminary analysis suggests five themes:

Patients recall little communication about PA: Few patients recalled PA commu- nication during their appointment, although some discussed general lifestyle and some recalled discussions about PA or exercise in previous consultations. Appointments focus on medical management: Patients mainly recalled discussing medical management of IA. Some were asked about general health and support needs, but assumed health professionals were interested in disease status and medication rather than lifestyle and wellbeing.

Patients are uncertain as to what constitutes PA: Some patients seemed confused by what constituted discussion about PA. Different interpretations were reported, with many participants referring to condition specific exercises rather than lifestyle PA. Few patients described PA as general movement or everyday activity. Patients would like to discuss PA: Few patients initiated conversation about PA, but many would welcome PA communication from any health professional with appropriate knowledge. Several patients felt most comfortable discussing PA with a member of their rheumatology team rather than a non-specialist. A small number felt the rheumatologist's role related to medical management rather than lifestyle advice. Preferences for communication style included a direct, honest approach using lay language. It was felt that health professionals could do more to ask about and encourage PA, reassure about PA safety, and offer individualized support to enable appropriate PA.

Perceived barriers to undertaking PA must be considered: Some patients perceived barriers to PA, such as lack of knowledge and skills for PA, lack of time, work commitments and IA flares. It was felt important to acknowledge that it can be difficult to apply lifestyle changes in practice.

Conclusions: IA patients recalled little discussion about PA in routine rheumatology consultations. Patients may interpret questions about general health and exercise as relating to their disease status and joint function rather than to lifestyle or PA. Health professionals should ask direct questions about lifestyle PA and offer non-didactic, individualized advice and support. Further research is needed to understand PA communication from the perspective of rheumatology health professionals, including identification of opportunities and challenges for implementing effective communication.

Disclosure of Interest: None declared

DOI: 10.1136/annrheumdis-2017-eular.6866

\section{SAT0770-HPR IS THE DUAL - TASK TRAINING BENEFICIAL ON THE CLINICAL OUTCOMES OF ELDERLY?}

Y. Aydoğdu ${ }^{1}$, O. Aydoğdu ${ }^{2}$, S.H. İnal ${ }^{3}{ }^{1}$ Department of Physiotherapy and Rehabilitation, Haydarpaşa Numune Hospital; ${ }^{2}$ Department of Physiotherapy and Rehabilitation, Health Sciences Faculty, Marmara University; ${ }^{3}$ Department of Physiotherapy and Rehabilitation, Health Sciences Faculty, Bahcesehir University, Istanbul, Turkey

Background: The difficulties of elderly in performing two different tasks have been well documented (1). It has been reported that aging is associated with a decline in attention abilities and dual-task performances. Improving the ability to perform two different tasks simultaneously could therefore have significant impacts in the prevention of adverse outcomes associated with aging (2).

Objectives: Regarding specific dual-task training, recent studies have demonstrated its efficacy in various populations such as the elderly and individuals with neurological diseases, with the most notable improvements in gait. However, there is no evidence in the literature on the effects of this training on balance and clinical outcomes evaluated independently among elderly. Thus, considering the positive results of specific dual-task training obtained in other populations, we conducted a randomized trial to study the efficacy of an eight - week dual-task training program adjunct to conventional physiotherapy compared to a conventional physiotherapy program on balance, mobility, functional independence, fear of falling, functional capacity in elder individuals.

We hypothesized that, dual-task training is more effective at improving balance and clinical outcomes than conventional physiotherapy in elder individuals.

Methods: Fifty-five elderly were participated in our study. Balance, mobility, functional independence, fear of falling was measured with Berg Balance Scale, Rivermead Mobility Index, Functional Independence Measurement, and Fall Efficacy Scale-International, respectively. Functional capacity was assessed with Timed Up\&Go, Chair Sit\&Stand. Cases were divided into two different groups. Conventional Physiotherapy was applied in control group, and besides the same protocol, dual-task training was applied 5 days for 8 weeks in the intervention group. The dual-task training consisting of the cognitive activities included digit span, spelling words, stroop test, image description, counting, description of daily activities and routines was applied during the gait exercises. All of the assessments procedures were performed again after the treatment.

Results: There were statistically significant improvements in measures of balance, mobility, functional independence, fear of falling, and functional capacity between pre- and post - treatment in both groups $(p<0.05)$. A significant difference was found in balance and mobility in favour of the intervention group $(\mathrm{p}=0.028)$.

Conclusions: Based on our findings, we found that dual-task training applied as an adjunct to conventional physiotherapy was superior to conventional physiotherapy on balance and mobility parameters. From this point of view, dual-task training should be considered as part of the rehabilitation process of elderly, although until now no guidelines have been defined for this type of intervention.

References:

[1] Bherer L, Kramer AF, Peterson MS, Colcombe S, Erickson K, Becic E. Transfer 